\title{
Should Canadian patients look forward to aducanumab for Alzheimer disease?
}

\author{
Jennifer A. Watt MD PhD, Roger Marple, Brenda Hemmelgarn MD PhD, Sharon E. Straus MD MSc
}

Cite as: CMAJ 2021 September 13;193:E1430-1. doi: 10.1503/cmaj.211134

I n Canada, 190000 people are living with mild Alzheimer-type dementia and 532000 to 799000 people are living with amnestic mild cognitive impairment. ${ }^{1-4}$ Developing treatments for Alzheimer disease is challenging, with few effective therapies that only target symptoms. Although the US Food and Drug Administration (FDA) approved aducanumab on June 7, 2021 - the first new treatment for Alzheimer disease since 2003 its approval was controversial. ${ }^{5}$

Aducanumab is a human monoclonal immunoglobulin (Ig) G1 antibody that selectively targets amyloid- $\beta$ aggregates (including soluble oligomers and insoluble fibrils) and clears amyloid plaques from the brain via a microglia-mediated phagocytic process. ${ }^{6}$ Biogen sponsored ENGAGE (NCT02477800) and EMERGE (NCT02484547), which are both phase 3, parallel group, placebocontrolled randomized trials involving aducanumab. Both trials enrolled patients aged 50 to 85 years with mild cognitive impairment or mild dementia caused by Alzheimer disease who had a positron emission tomography (PET) scan that showed brain amyloid pathology. Patients were randomized to receive lowdose aducanumab, high-dose aducanumab or placebo for 18 months. Because of concern that carriers of apolipoprotein $\mathrm{E}$ (ApoE) episilon-4 could be at higher risk of developing amyloidrelated imaging abnormalities (ARIA), than noncarriers, Biogen stratified patients by ApoE episolon-4 status. ${ }^{7}$ For example, ApoE epsilon-4 carriers who were randomized to high-dose aducanumab received $6 \mathrm{mg} / \mathrm{kg}$ and noncarriers received $10 \mathrm{mg} / \mathrm{kg}$ after a 24-week titration period. ${ }^{8}$ However, the protocol was amended after a phase $1 \mathrm{~b}$ randomized trial (completed after phase 3 trials began) established that it was safe to administer a $10 \mathrm{mg} / \mathrm{kg}$ dose to ApoE epsilon-4 carriers randomized to the high-dose aducanumab group. ${ }^{8}$

ENGAGE and EMERGE were both terminated for futility on Mar. 21, 2019, after a prespecified interim analysis, but Biogen successfully argued in support of aducanumab's potential efficacy as a disease-modifying therapy based on posthoc analyses, and the FDA approved aducanumab for use in people with mild cognitive impairment or mild dementia caused by Alzheimer disease. ${ }^{5,8}$ The FDA's approval of aducanumab went against the recommendation of its independent Peripheral and Central Nervous System Drugs advisory committee, which recommended to the

\section{Key points}

- The recent approval of aducanumab by the US Food and Drug Administration was controversial.

- Both phase 3 trials for aducanumab were terminated for futility after a prespecified interim analysis, and the clinical significance of their findings remains unclear.

- Patients and clinicians are worried about aducanumab's uncertain benefit, treatment burden (e.g., needing intravenous infusions of aducanumab every 4 weeks), safety and cost.

- Currently, aducanumab is not approved for use in Canada, but it has been submitted to Health Canada for review.

FDA that aducanumab not be approved because of lack of efficacy. Some trial analyses were conducted in close collaboration with the FDA, which may be perceived to have influenced the FDA's decision. ${ }^{5,8}$

The FDA's approval of aducanumab was also atypical because it was approved through the Accelerated Approval pathway, which is intended for drugs that are for a "serious or life-threatening illness that may provide meaningful therapeutic benefit over existing treatments when the drug is shown to have an effect on a surrogate endpoint that is reasonably likely to predict a clinical benefit to patients and there remains some uncertainty about the drug's clinical benefit." ${ }^{5}$ Although aducanumab resulted in statistically significant time- and dose-dependent reductions in brain amyloid plaque compared with placebo, as measured by PET and other biomarkers in EMERGE and ENGAGE, the association between reduced amyloid levels and improvement in cognitive outcomes is uncertain. ${ }^{8,9}$ Furthermore, the accumulation of amyloid- $\beta$ (i.e., the amyloid hypothesis) may not be the sole cause of Alzheimer disease, which makes its appropriateness as a surrogate end point questionable., 8

Reliance on a surrogate end point, as opposed to primary and secondary clinical outcomes, was also important because of the uncertain clinical significance of clinical outcomes seen in the EMERGE trial. In the ENGAGE trial, neither statistically nor clinically significant treatment differences were found for primary or secondary clinical outcomes. For example, the minimum clinically important difference (i.e., the threshold above which clinicians, 
patients and researchers perceive a difference on an outcome scale) on the Clinical Dementia Rating-Sum of Boxes score (the primary outcome) is 1 to 2 points, ${ }^{10}$ but the difference in scores between high-dose aducanumab and placebo groups was -0.39 points $(p=0.0120)$ in EMERGE. ${ }^{8}$ Further, the minimum clinically important difference on the Mini-Mental State Examination is 1 to 3 points, ${ }^{10}$ but in the EMERGE trial, the difference between high-dose aducanumab and placebo groups in this secondary outcome was only 0.6 points $(p=0.0493){ }^{8}$

Patients in Canada living with mild cognitive impairment or mild dementia caused by Alzheimer disease hope for a drug that can provide them with clinically meaningful benefit, but it is unclear if aducanumab is this drug. They rightly worry about treatment burden (i.e., aducanumab is administered by intravenous infusion every 4 weeks and requires magnetic resonance imaging of the brain to monitor for ARIA) and potential adverse events. ${ }^{8}$ Pooled safety data from EMERGE and ENGAGE showed that, compared with the placebo group, the $10 \mathrm{mg} / \mathrm{kg}$ aducanumab group had higher rates of ARIA of edema or effusion (35.0\% v. $2.7 \%$ ), ARIA of microhemorrhages and hemosiderin deposits ( $20.5 \%$ v. $6.5 \%)$, headache ( $20 \%$ v. $15 \%)$ and falling $(15 \%$ v. $12 \%) ; 10 \%$ of patients in the group receiving aducanumab at a dose of $10 \mathrm{mg} / \mathrm{kg}$ had symptomatic ARIA. ${ }^{8}$ Further, more patients in the aducanumab $10 \mathrm{mg} / \mathrm{kg}$ group stopped treatment because of an adverse event compared with placebo (8.8\% v. $4.1 \%$ ), which was secondary to an increased incidence of ARIA in the aducanumab group. ${ }^{8}$ This high incidence of ARIA was observed despite trial eligibility being limited to patients taking acetylsalicylic acid at a dose of $325 \mathrm{mg} / \mathrm{d}$ or less (i.e., excluding patients using other antiplatelet or anticoagulant agents) and potential healthy user bias, whereby clinical trial participants are healthier and therefore at lower risk of adverse outcomes than patients who will receive the drug in real-world settings. Patients also worry about potential treatment-related costs and whether aducanumab will be accessible to all patients for whom it is potentially indicated. In the United States, Biogen has priced aducanumab at $\$ 56000$ per year; however, the fair annual price is $\$ 2500$ to $\$ 8300$ per year. ${ }^{11}$

Results from the EMERGE and ENGAGE trials have not yet been published in a peer-reviewed journal. Biogen recently withdrew a manuscript from the Journal of the American Medical
Association containing phase 3 clinical trial results for aducanumab. ${ }^{12}$ Currently, aducanumab is not approved for use in Canada, but it has been submitted to Health Canada for review. ${ }^{13}$ Based on available data, as physicians and researchers (J.A.W., B.H., S.E.S.), we would not recommend its approval for use in Canada; R.M., a patient partner, will await Health Canada's decision.

\section{References}

1. Yuan J, Maserejian N, Liu Y, et al. Severity distribution of Alzheimer's disease dementia and mild cognitive impairment in the Framingham Heart Study. J Alzheimers Dis 2021;79:807-17.

2. Jansen WJ, Ossenkoppele R, Knol DL, et al. Prevalence of cerebral amyloid pathology in persons without dementia: a meta-analysis. JAMA 2015;313:1924-38.

3. Petersen RC, Lopez O, Armstrong MJ, et al. Practice guideline update summary: mild cognitive impairment: report of the Guideline Development, Dissemination, and Implementation Subcommittee of the American Academy of Neurology. Neurology 2018;90:126-35.

4. Prevalence and monetary costs of dementia in Canada. Toronto: Alzheimer Society of Canada; 2016.

5. Cavazzoni P. FDA's decision to approve new treatment for Alzheimer's disease [news release]. Silver Spring (MD): US Food and Drug Administration; 2021 June 7.

6. Sevigny J, Chiao P, Bussiere T, et al. The antibody aducanumab reduces betaamyloid plaques in Alzheimer's disease. Nature 2016;537:50-6.

7. Sperling R, Salloway S, Brooks DJ, et al. Amyloid-related imaging abnormalities in patients with Alzheimer's disease treated with bapineuzumab: a retrospective analysis. Lancet Neurol 2012;11:241-9.

8. Combined FDA and Biogen briefing information for the November 6, 2020 meeting of the Peripheral and Central Nervous System Drugs Advisory Committee. Silver Spring (MD): US Food and Drug Administration; 2020 Nov. 6. Available: https://www.fda.gov/media/143502/download (accessed 2021 June 16).

9. Ackley SF, Zimmerman SC, Brenowitz WD, et al. Effect of reductions in amyloid levels on cognitive change in randomized trials: instrumental variable metaanalysis. BMJ 2021;372:n156.

10. Andrews JS, Desai U, Kirson NY, et al. Disease severity and minimal clinically important differences in clinical outcome assessments for Alzheimer's disease clinical trials. Alzheimers Dement (N Y) 2019;5:354-63.

11. ICER issues statement on the FDA's approval of aducanumab for Alzheimer's disease [press release]. Boston: Institute for Clinical and Economic Review; 2021 June 17. Available: https://icer.org/news-insights/press-releases/ icer-issues-statement-on-the-fdas-approval-of-aducanumab-for-alzheimers -disease/ (accessed 2021 June 17).

12. Herman B. Biogen pulled Aduhelm paper after JAMA demanded edits. AXIOS.com 2021 July 27. Available: https://www.axios.com/biogen-jama-aduhelm-clinical -trial-results-publish-fc7c2876-a684-4bfc-8462-4165f57d735a.html (accessed 2021 July 28).

13. Notice regarding Biogen's disclosure about the submission of marketing authorization applications in Brazil, Canada, Australia, and Switzerland for Aducanumab for Alzheimer's disease [news release]. Eisai Global 2021 Apr. 23.
Competing interests: Roger Marple reports unpaid committee participation with AGEWELL, the Alzheimer's Society of Canada, the Alzheimer's Society of Alberta and Northwest Territories, the World Health Organization and Dementia Advocacy Canada. No other competing interests were declared.

This article has been peer reviewed.

Affiliations: Knowledge Translation Program (Watt, Straus), Li Ka Shing Knowledge Institute, St. Michael's Hospital; Division of Geriatric Medicine, Department of Medicine (Watt, Straus),
University of Toronto, Toronto, Ont.; Patient partner (Marple), Medicine Hat, Alta.; Faculty of Medicine and Dentistry (Hemmelgarn), University of Alberta, Edmonton, Alta.; Institute of Health Policy, Management and Evaluation (Straus), Dalla Lana School of Public Health, University of Toronto, Toronto, Ont.

Contributors: All authors contributed to the manuscript's conception and design. Jennifer Watt wrote the first manuscript draft. All authors provided feedback, approved the final manuscript version and agreed to be accountable for all aspects of this work.
Content licence: This is an Open Access article distributed in accordance with the terms of the Creative Commons Attribution (CC BY-NC-ND 4.0) licence, which permits use, distribution and reproduction in any medium, provided that the original publication is properly cited, the use is noncommercial (i.e., research or educational use), and no modifications or adaptations are made. See: https://creativecommons. org/licenses/by-nc-nd/4.0/

Correspondence to: Jennifer Watt, jennifer. watt@mail.utoronto.ca 